\title{
Automatic Postural Responses Are Delayed by Pyridoxine-Induced Somatosensory Loss
}

\author{
Paul J. Stapley, ${ }^{1}$ Lena H. Ting, ${ }^{1}$ Manuel Hulliger, ${ }^{2}$ and Jane M. Macpherson ${ }^{1}$ \\ ${ }^{1}$ Neurological Sciences Institute, Oregon Health and Science University, Beaverton, Oregon 97006-3499, and \\ 2Department of Clinical Neurosciences, University of Calgary, Alberta, T2N 4N1, Canada
}

Pyridoxine given in large doses is thought to destroy selectively the large-diameter peripheral sensory nerve fibers, leaving motor fibers intact. This study examined the effects of pyridoxineinduced somatosensory loss on automatic postural responses to sudden displacements of the support surface in the standing cat. Two cats were trained to stand on four force plates mounted on a movable platform. They were given pyridoxine (350 mg/kg, i.p.) on 2 successive days (0 and 1). Electromyographic (EMG) activity was recorded from selected hindlimb muscles during linear ramp-and-hold platform displacements in each of 12 directions at $15 \mathrm{~cm} / \mathrm{sec}$. In control trials onset latencies of evoked activity in hindlimb flexor and extensor muscles ranged from 40 to $65 \mathrm{msec}$ after the onset of platform acceleration. After injection the EMG latencies increased over

Automatic postural responses (APRs) are highly stereotyped patterns of electromyographic (EMG) activity in various muscles, triggered in response to sudden disturbances of balance. It is thought that somatosensory, vestibular, and visual inputs are integrated for assessing postural equilibrium (Horak and Macpherson, 1996). It is not clear, however, which sensory channel is responsible for triggering and shaping the rapid response to sudden movements of the support surface. Somatosensory or vestibular inputs or both could, in theory, trigger rapid postural reactions to sudden disturbances, whereas vision generally is regarded as being too slow (Horak and Macpherson, 1996).

Previously, somatosensation has been accorded a modulatory role in motor control. For example, goal-directed arm and leg movements and rhythmic movements can be performed without somatosensory feedback (Prochazka, 1996). Central pattern generators used to produce stepping and paw shakes also do not require sensory feedback (Grillner, 1975; Koshland and Smith, 1989), and patients with large-fiber peripheral neuropathy who lack cutaneous or proprioceptive inputs can rely on vision to guide multijoint arm movements (Sanes et al., 1985; Ghez et al., 1995; Larue et al., 1995). If somatosensory inputs are only facilitatory for posture as well, then vestibular afferents should be critical for the appropriate timing of postural responses. How-

Received Feb. 20, 2002; revised May 1, 2002; accepted May 1, 2002.

This work was supported by National Institutes of Health Grant NS29025. L.H.T. is an O'Donnell Foundation Fellow of the Life Sciences Research Foundation. We gratefully acknowledge the skilled technical assistance of Stephanie Jensen, Dr. Charles J. Russell, and Nancy R. Schuff.

Correspondence should be addressed to Dr. Jane M. Macpherson, Neurological Sciences Institute, Oregon Health and Science University, West Campus 505 Northwest 185th Avenue, Beaverton, OR 97006-3499. E-mail: macphers@ohsu.edu. Copyright (C) 2002 Society for Neuroscience $0270-6474 / 02 / 225803-05 \$ 15.00 / 0$ days, becoming two to three times longer than controls by day 7. Excursions of the body center of mass (CoM) in the direction opposite to that of platform translation were significantly greater at day 7 compared with controls, and the time at which the CoM subsequently reversed direction was delayed. Both animals were ataxic from day 2 onward. Histological analysis of cutaneous and muscle nerves in the hindlimb revealed a significant loss of fibers in the group I range. Our results suggest that large afferent fibers are critical for the timing of automatic postural responses to ensure coordinated control of the body CoM and balance after unexpected disturbances of the support surface.

Key words: posture and balance; EMG; cat; pyridoxine; somatosensory loss; peripheral neuropathy

ever, the removal of vestibular input does not alter the temporal or spatial pattern of APRs, even in the acute phase immediately after lesion and in the absence of vision (Inglis and Macpherson, 1995). Because vestibular input is not essential for triggering responses to support surface disturbance, somatosensation should be critical to balance control, although there is no direct evidence for this. In this study we investigated the role of somatosensory information in the response to unexpected support surface translations in the standing cat, using the neurotoxicity of pyridoxine as a tool.

Pyridoxine (vitamin $\mathrm{B}_{6}$ ) given in high doses produces selective large-fiber sensory loss in humans (Schaumburg et al., 1983), dogs (Hoover and Carlton, 1981), and rats (Krinke et al., 1978; Xu et al., 1989) and currently is being developed as a noninvasive technique of large-fiber deafferentation in the cat [Allum et al., 1998, their pp. 226-233; Hulliger et al. (2000)]. The mechanism of pyridoxine toxicity is unknown. However, histological studies in animals have shown that the toxic effects of pyridoxine are limited to the peripheral and central processes of large myelinated primary afferent fibers but that neurons of the CNS are not affected directly (Windebank et al., 1985; Xu et al., 1989). This study used pyridoxine to remove selectively the large-diameter somatosensory inputs in the cat. We provide clear new evidence that somatosensation is essential to the correct timing of APRs to support surface translations.

\section{MATERIALS AND METHODS}

Two adult female cats (Wo and $\mathrm{Kn}$ ) weighing 3.2 and $4 \mathrm{~kg}$, respectively, were trained by using positive reinforcement to stand quietly on four miniature triaxial force plates mounted on a movable platform. The animals were required to distribute their weight equally between the left and right sides. Details of equipment and training procedures have been 
described previously (Macpherson et al., 1987). The position of the force plates was adjusted to each cat's "preferred" stance distance, determined from its natural fore-hindpaw distance while standing on the floor. Stance distances were $26 \mathrm{~cm}$ along the anteroposterior axis and $8 \mathrm{~cm}$ mediolateral for both cats and were conserved for the entire duration of the experiments. Once trained, the animals were implanted with chronic indwelling electrodes into left forelimb and hindlimb muscles under general anesthesia (Saffan), using aseptic techniques (for details, see Macpherson, 1988). Muscles assessed for this report included gluteus medius (GLUT), anterior sartorius (SRTA), middle biceps femoris (BFMM), lateral gastrocnemius (LGAS), soleus (SOL), posterior semimembranosus (SEMP), and anterior semimembranosus (SEMA).

A hydraulically driven force platform interfaced to a computer for data acquisition and control delivered linear translations of the entire support surface in 12 equally spaced directions in the horizontal plane. An initial data acquisition period of $300 \mathrm{msec}$ was followed by a ramp-and-hold platform displacement (55 mm amplitude; mean peak velocity, $15 \mathrm{~cm} /$ $\mathrm{sec})$. The total data collection period was $3 \mathrm{sec}$, after which the platform returned to its initial position. Within each session five trials were collected in each direction. Perturbations were given when animals were standing quietly with their heads facing forward and their weight distribution met the training criteria.

Once all control data were collected, each cat was given an injection of pyridoxine $(350 \mathrm{mg} / \mathrm{kg}$, i.p.) in the evening on each of 2 successive days (days 0 and 1, respectively). Postural data were collected every day until day $8(\mathrm{Wo})$ or $13(\mathrm{Kn})$. One experimenter stood behind the animal to prevent falls during loss of balance.

Increases in EMG activity after translation were deemed significant when the amplitude exceeded the mean background by $>2.5$ SD for a minimum of $10 \mathrm{msec}$. The latency of the EMG burst was specified as the time when the signal exceeded mean background $+0.3 \mathrm{SD}$. Only latencies of $<100 \mathrm{msec}$ were considered for further analysis in the control data, whereas the postpyridoxine series included latencies $\leq 300 \mathrm{msec}$ because responses were delayed considerably. This report focuses on the differences between control data and those collected at day 7, when both cats showed the greatest delay in postural response latencies. Response latencies did not return to control values before the deaths of the animals.

For each direction the position of the center of mass (CoM) in the horizontal plane was calculated from force-time curves (for details, see Macpherson, 1994). Briefly, horizontal plane forces were summed across the four paws and divided by the animal's mass to give CoM acceleration along each axis, lateral and longitudinal. Traces were integrated twice to obtain position, with the mean position of the center of pressure during quiet stance providing the initial CoM position.

We quantified the effects of pyridoxine by analyzing changes in EMG latency with respect to platform onset, and the trajectory of the CoM, between control data and those collected at day 7. A one-way ANOVA for unequal numbers was used to analyze differences between days for (1) latency of EMG activation, (2) maximum CoM displacement, and (3) time of maximum CoM displacement.

We monitored the severity of motor deficits associated with pyridoxine deafferentation by recording various reflexes. First, stretch reflexes in the gastrocnemius muscle were elicited by tapping briskly on the Achilles tendon with a small Plexiglas bar with a curved cutout that fit over the tendon. The bar was instrumented with a relay that delivered one pulse for each tap. EMGs and the pulse were recorded on-line at 3000 samples/ $\mathrm{sec}$ for $100 \mathrm{msec}$. Ten trials of 40 taps were recorded during control sessions and every day after pyridoxine. Second, reflex responses evoked by free fall were recorded during sudden dropping of the animal. The cat was placed in a bag, and vision was occluded with a muzzle that covered the eyes but did not obstruct breathing. A linear accelerometer was attached to the connector on the head. EMGs of forelimb and hindlimb muscles and head acceleration were recorded on-line while animals were suspended $2 \mathrm{~m}$ above the floor and dropped into the arms of a second experimenter who caught them with a gentle decelerating motion.

After the completion of data collection each cat was anesthetized deeply and perfused intracardially for harvesting of peripheral nerves. Control material came from an intact animal not used in this series of studies. Two animals (control, Kn) were heparinized and perfused with $4 \%$ paraformaldehyde, followed by $5 \%$ glutaraldehyde, both in $0.15 \mathrm{M}$ phosphate buffer; the third (Wo) was perfused with heparinized Ringer's, followed by $3 \%$ paraformaldehyde plus $2 \%$ glutaraldehyde in $0.15 \mathrm{M}$ phosphate buffer. Cutaneous and muscle nerves were harvested from both sides of the body immediately after fixation, placed in glutaraldehyde, and subsequently stored in $0.15 \mathrm{M}$ cacodylate buffer. For consis- tency across animals, sampling locations were based on anatomical landmarks. Nerves were postfixed in $2 \%$ osmium tetroxide and infiltrated and embedded in Spurr epoxy resin. They were sectioned transversely at 1 $\mu \mathrm{m}$ thickness and stained with toluidine blue. Tissue sections were viewed with a light microscope, digitized, and then analyzed with the MetaMorph image analysis system (Universal Imaging, West Chester, PA). The cross-sectional area of all myelinated fibers was measured, and fiber diameters were estimated assuming a circular cross section. In this study the data from the distal saphenous (cutaneous) and the medial gastrocnemius (mixed) nerves will be reported. In all cats the saphenous nerve was harvested $20 \mathrm{~mm}$ proximal to the ankle joint. The medial gastrocnemius nerve was taken $10 \mathrm{~mm}$ from its insertion into the muscle.

\section{RESULTS}

Clear signs of ataxia appeared on day 2 in the form of occasional foot misplacement or tilting during gait. Symptoms progressed through days 3 and 4 such that cats often slipped or were unbalanced when turning during locomotion. By day 5 both animals staggered, showing poor control of the trunk, but they did not lose the ability to walk independently. However, they did have difficulty balancing independently on the platform and required almost continuous lateral stabilization. The need for assistance may have been attributable, in part, to a sense of insecurity on the elevated surface, which is relatively small. On day 7, animals had accommodated sufficiently to be able to stand without assistance during data collection. Symptoms of ataxia did not change after day 7 up to the time of death.

On the platform both cats were able to remain balanced during and after translation on days 1 and 2. On days 3 and 4 they stepped or fell during translations for $\sim 25 \%$ of trials. By day 4 (Wo) or day $5(\mathrm{Kn})$ the cats required lateral stabilization while standing on the platform during both quiet stance and translations, although they could maintain independent weight support. Although the need for lateral stabilization on the platform was transient, nevertheless translations evoked falls or stepping for $40-45 \%$ of trials even on day 7 . We present data for day 7 because the animals did not require external support, and so the ground reaction forces accurately reflected all of the forces acting on the animal.

\section{EMG responses to unexpected platform displacements are delayed significantly after pyridoxine administration}

Onset latencies were increased significantly by day 7 after injection (see Table 1), although directional tuning of muscles remained similar to controls. Figure 1 shows typical EMG activity of selected hindlimb muscles before and after pyridoxine administration. During control trials the latencies ranged from 40 to 65 msec after the onset of platform acceleration, whereas after pyridoxine the latencies ranged from 91 to $222 \mathrm{msec}$. This represents a twofold to threefold increase with respect to control values. Increases were highly statistically significant for all of the hindlimb muscles that were studied. In addition, the timing and amplitude of evoked activity were considerably more variable both within and across muscles for each cat (Fig. 1).

\section{Delayed automatic postural response impairs balance control}

The fact that both animals stepped or fell at day 7 indicates they had difficulty controlling their balance. Figure $2 A$ shows typical examples of horizontal CoM trajectories before and after pyridoxine administration for both cats in one selected direction of translation $\left(240^{\circ}\right)$. During control trials (Fig. $2 A$, black trajectories) the CoM moved opposite to the direction of translation, reversed trajectory, and returned close to its original position. 


\begin{tabular}{|c|c|c|c|c|c|c|}
\hline & BFMM & GLUT & SRTA & SEMP & SEMA & LGAS \\
\hline \multicolumn{7}{|l|}{ Cat Wo } \\
\hline Control & $54(7)$ & $48(5)$ & 49 (7) & $52(7)$ & & $50(5)$ \\
\hline Day 7 & $150(44)^{* *}$ & $106(60)^{* *}$ & $120(35)^{* *}$ & $139(18)^{* *}$ & & $132(44)^{* *}$ \\
\hline \multicolumn{7}{|l|}{ Cat Kn } \\
\hline Control & $47(7)$ & $40(9)$ & $65(25)$ & $52(16)$ & 45 (11) & \\
\hline Day 7 & $98(24)^{* *}$ & $124(70)^{* *}$ & $222(30)^{* *}$ & $91(9)^{*}$ & $139(60)^{* *}$ & \\
\hline
\end{tabular}

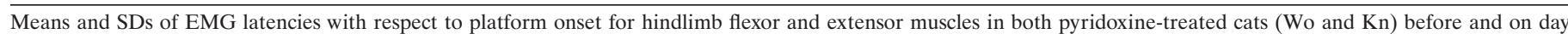

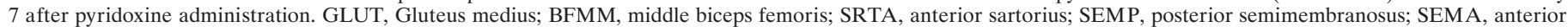
semimembranosus; LGAS, lateral gastrocnemius. Significant difference from control values at $* p<0.05$ and $* * p<0.01$, respectively.

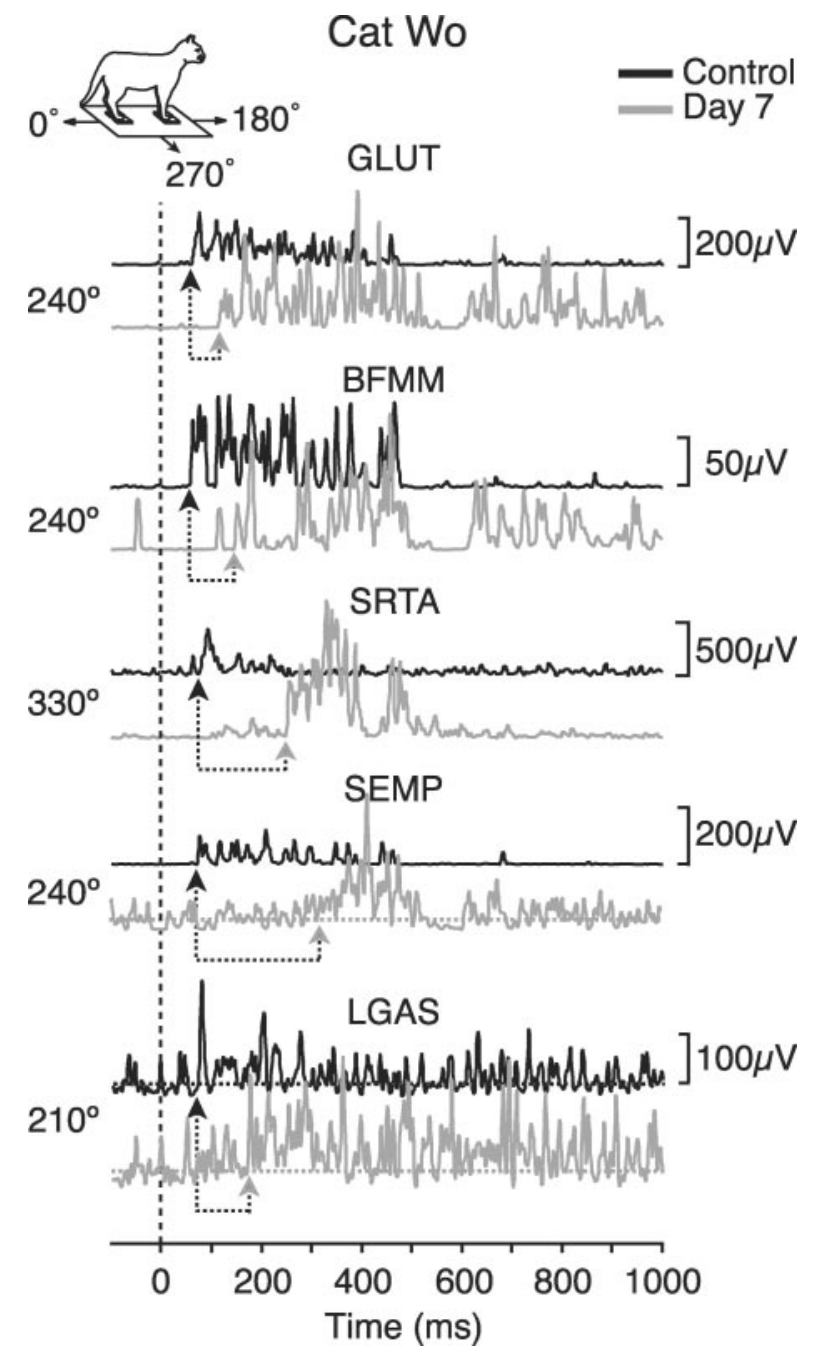

Figure 1. Representative averaged EMG activity of hindlimb muscles for cat Wo before (black) and on day 7 after pyridoxine (gray). The translation directions that are illustrated are those for which each muscle was activated maximally in the control condition. The dashed vertical line indicates the onset of platform acceleration. For muscles SEMP and LGAS the baseline EMG activity is indicated by horizontal dotted lines to clarify initial burst onset. Under each trace, arrows indicate response onset during control trials and at day 7. GLUT, Gluteus medius; BFMM, middle biceps femoris; SRTA, anterior sartorius; SEMP, posterior semimembranosus; $L G A S$, lateral gastrocnemius. Inset shows coordinate reference for direction of translation.

\section{A. Horizontal plane center of mass trajectory}
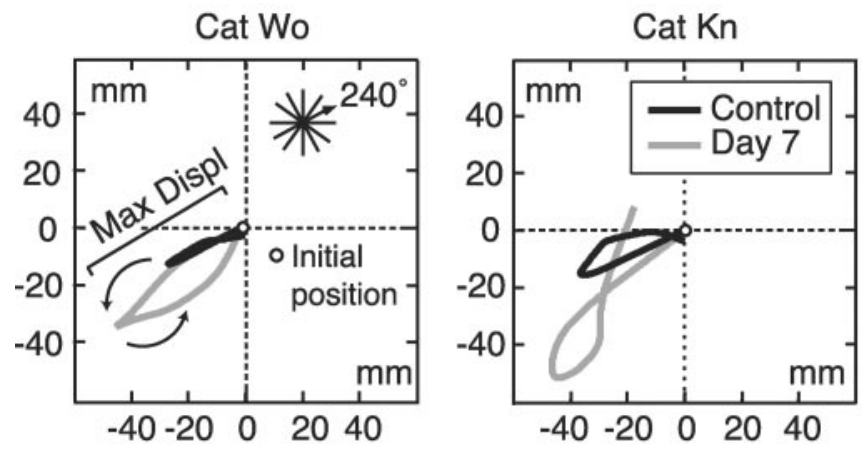

\section{B. Max Displ}

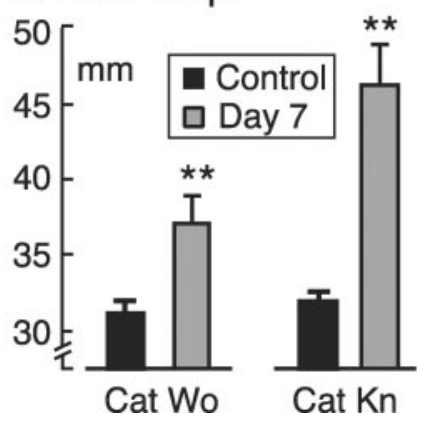

C. Time of Max Displ

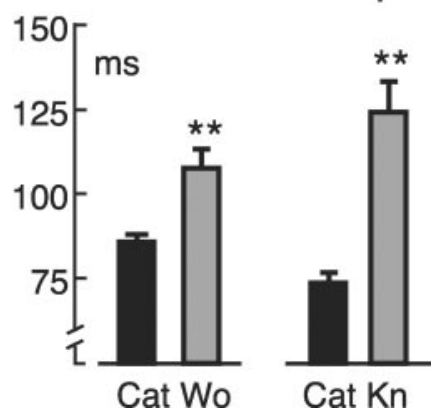

Figure 2. A, Representative horizontal plane center of mass (CoM) trajectories for each cat in control (black) and postpyridoxine (gray) conditions for one selected direction $\left(240^{\circ}\right)$. For comparison, control and postpyridoxine trajectories are aligned to the same initial position (open circles). $B$, Amplitude of maximum initial displacement of the CoM from its origin (see region labeled Max Displ in $A$ ). $C$, Time of maximum displacement with respect to the onset of platform translation. Error bars indicate SE. **Significantly different from control values $(p<0.001)$.

After pyridoxine (Fig. 2A, gray trajectories) the CoM of both cats traveled a greater distance in the direction opposite to translation before reversing toward the initial position. The CoM of cat $\mathrm{Kn}$ in particular often failed to return close to its initial position. The maximum displacement of the CoM was significantly greater after pyridoxine (Fig. 2B). Moreover, the time of maximum displacement at which the CoM reversed direction was significantly longer for both cats (Fig. 2C).

\section{Tendon tap reflex responses are eliminated by pyridoxine, but reflex responses to dropping remain intact}

Ankle extensor muscles (LGAS and SOL for Wo and Kn, respectively) showed early (10 msec latency) and brisk responses to 


\section{Saphenous nerve}
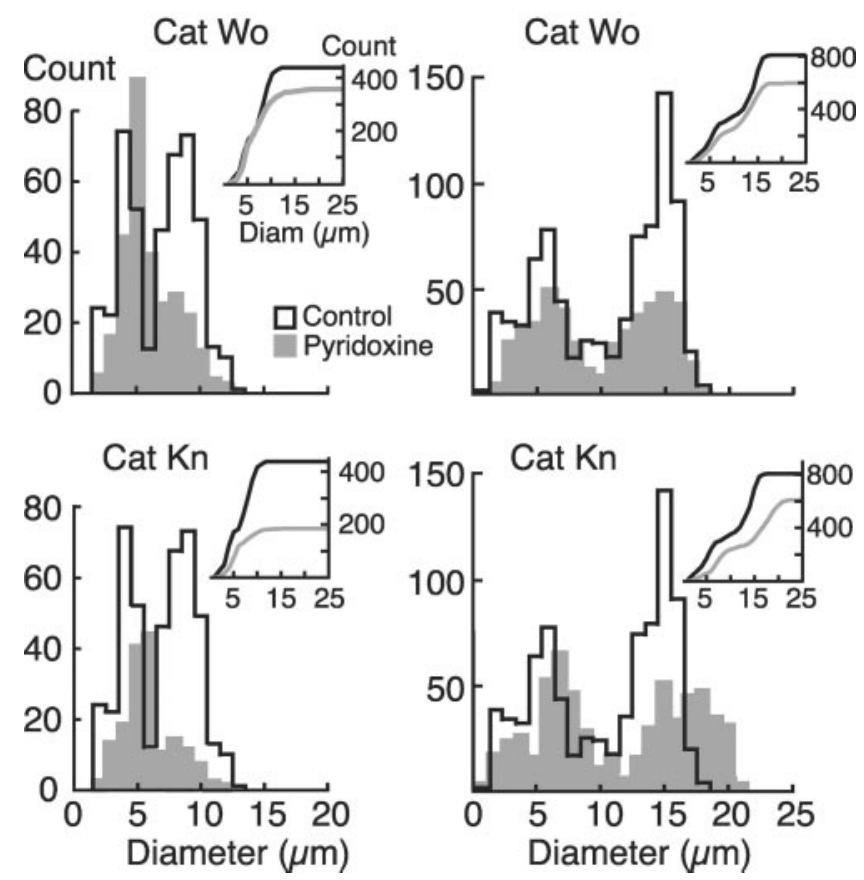

Figure 3. Frequency versus diameter of all myelinated nerve fibers for saphenous (cutaneous) and medial gastrocnemius (mixed motor and sensory) nerves for both pyridoxine cats (gray) and one control (black). In each, the main plot shows absolute counts for diameters in $1 \mu \mathrm{m}$ bins, and the inset at top right shows cumulative frequency plots of the same data. The saphenous nerve was sampled $2 \mathrm{~cm}$ above the ankle and the gastrocnemius at $1 \mathrm{~cm}$ from its entry point to the muscle.

tapping of the Achilles tendon in control trials, but responses declined gradually and disappeared after pyridoxine administration. Between days 1 and 3 the amplitude of tap response decreased, and by day 5 both cats failed to show any tendon tap responses at all. This absence of response persisted until each cat was killed.

The reflex response to sudden dropping did not change after pyridoxine administration. Animals attained free fall $\sim 200 \mathrm{msec}$ after the beginning of release and remained in free fall for $\sim 250$ msec. Forelimb and hindlimb muscles were activated $\sim 30-50$ msec before the attainment of free fall in control trials. On day 8 both cats demonstrated similar latency and amplitude of muscular responses to drops, as in the control trials. These reflex responses are presumed to originate in the otoliths of the vestibular system (Watt, 1976). Post-rotatory nystagmus also could be elicited both before and after pyridoxine administration. These results suggest that vestibular function likely was not impaired by pyridoxine intoxication.

\section{Histological quantification of the effects of pyridoxine}

Both saphenous and gastrocnemius nerves in the pyridoxinetreated animals showed significant loss or damage of fibers in the range above $7 \mu \mathrm{m}$ in diameter. Many large fibers showed signs of degeneration, including empty myelin profiles and shriveled axons with heavily stained, clumped inclusions. The diameters of the normal-appearing fibers were used to generate frequency histograms and cumulative plots in control and pyridoxinetreated animals. Fibers of the normal saphenous nerve had two peaks in the frequency distribution, at $\sim 4$ and $9 \mu \mathrm{m}$ (Fig. 3, left, open histograms). The treated animals showed a marked decrease in the numbers of fibers in the larger peak (Fig. 3, left, gray filled histograms). The cumulative distribution plots diverged at $\sim 7$ $\mu \mathrm{m}$, indicating that the loss of fibers was predominantly above this value, which includes both group I and some group II fibers.

The medial gastrocnemius nerve of the control cat showed two peaks in fiber diameters at $\sim 6$ and $15 \mu \mathrm{m}$ (Fig. 3, right). As with the saphenous nerve, there was a significant loss of fibers predominantly in the large-diameter range. Note that many of the remaining large-diameter fibers are efferents from $\alpha$-motoneurons, which remain unaffected by pyridoxine. The fiber counts suggest that the saphenous nerve may have been affected somewhat more severely than the gastrocnemius, with losses in fibers $>7 \mu \mathrm{m}$ as opposed to $\sim 10 \mu \mathrm{m}$. More data are needed to confirm this observation, but there is some evidence that the conduction velocity of different afferent pathways may be affected differentially by pyridoxine (Schaeppi and Krinke, 1985).

\section{DISCUSSION}

In summary, pyridoxine intoxication induced an increase in latency of APRs resulting in impairment of the balance control system and was accompanied by a loss of large-diameter sensory afferent fibers.

\section{Somatosensory information is critical for the timing of} automatic postural responses

It is believed that the characteristics of APRs are shaped by a combination of somatosensory, vestibular, and visual inputs (Horak and Macpherson, 1996). APRs are rapid muscular responses that restore balance after unexpected disturbances. They are highly stereotyped, with a fixed latency in the cat between 45 and $60 \mathrm{msec}$ after the onset of the disturbance. The amplitude of the initial EMG burst reflects the direction and velocity of the disturbance (Diener et al., 1988; Macpherson, 1988).

Our results show that APRs were delayed when large somatosensory afferent fibers were damaged after pyridoxine administration. In contrast, the timing of APRs was unaffected by loss of vestibular information after bilateral labyrinthectomy, even when vision was absent (Inglis and Macpherson, 1995). We suggest, therefore, that large-diameter rapidly conducting somatosensory afferents are critical for the appropriate timing of balance responses, and visual and vestibular systems together are not able to compensate for delays in latency, at least during the period that was studied. These data support the hypothesis (Inglis et al., 1994) that postural delays in patients with diabetic peripheral neuropathy are attributable primarily to somatosensory conduction delays, although nerve conduction velocities were reduced for both sensory (afferent) and motor (efferent) nerves.

\section{Delays in response have a significant effect on balance control}

The timing of the APR is critical to maintaining balance, and it is likely that there is only a limited time period in which the response must occur to maintain control of the CoM. The role of the APR after a perturbation is to decelerate the CoM and return it to a position that ensures the maintenance of balance. After pyridoxine the increase in response latency was associated with a larger displacement of the CoM away from the original position and a longer time to reverse trajectory. Behaviorally, the animals were ataxic and often stepped or fell during translations, indicating that the effect on the CoM trajectory had a significant impact on balance control.

The increased variability in amplitude and timing of the EMG responses was accompanied by increased variability in CoM tra- 
jectory. The relationship between muscle activation and CoM motion in the ataxic cat is difficult to determine, in part because the mechanical effect of the imposed disturbance is more pronounced at the time the muscles are finally activated and the initial passive motion of the body is likely more variable. More subjects are required to assess adequately this question of variability in evoked response.

A previous study (Inglis and Macpherson, 1995) showed that vestibular loss produced ataxia that is similar in appearance to that of the pyridoxine cats, but the underlying cause is different. In the vestibular-loss animals the latency of the APR was normal, but the amplitude was increased. During translation the CoM initially moved opposite to the perturbation, but the displacement was shorter than in controls and reversed direction earlier in time, unlike the pyridoxine cats in which the CoM moved further and reversed later. The return phase in the vestibular loss animals was characterized by a large overshoot with subsequent oscillation about the final position. In other words, the hypermetric response resulted in overbalancing and increased body sway after a perturbation. Thus, ataxia can result from either abnormal timing or abnormal amplitude of automatic postural responses.

\section{Triggering of the early postural response}

Which fibers of the somatosensory system are responsible for triggering the early postural response? Our histological analysis showed that pyridoxine affected peripheral nerve fibers mainly in the group I range $(12-22 \mu \mathrm{m})$ but also some fibers in the group II range $(5-12 \mu \mathrm{m})$ (Boyd and Davey, 1968). Group I fibers innervate muscle spindle primary receptors that signal velocity of muscle stretch and Golgi tendon organs that signal changes in muscle force. Fibers from large cutaneous mechanoreceptors also may have diameters in the lower end of the group I range. Group II fibers innervate spindle secondaries and cutaneous receptors, among others. Because the most profound loss was in the group I range, it may be that the rapidly conducting fibers of this group are most accountable for the early timing of the postural response to unexpected support surface translations. Muscle receptors are more likely to be the trigger than cutaneous mechanoreceptors in the paw pads, because studies have shown that blocking afferents from the foot in humans does not delay the timing of balance responses to perturbation (Diener et al., 1984; Do et al., 1990).

In a recent study those patients with loss of large-diameter sensory and motor fibers also showed delays of the postural response, in this case to platform rotation (Nardone et al., 2000). However, the authors attributed the delay to efferent, rather than afferent, slowing and suggested that it was group II spindle afferents that triggered the postural response. In our study the remaining group II fibers were not sufficient to evoke a postural response with the normal timing, although they may have contributed to triggering of the response that was delayed. Although the question remains of which sensory receptor type is responsible for triggering APRs, it is clear that somatosensory afferents are critical to the timing of postural responses to sudden disturbances.

\section{REFERENCES}

Allum JHJ, Bloem BR, Carpenter MG, Hulliger M, Hadders-Algra M (1998) Proprioceptive control of posture: a review of new concepts. Gait Posture 8:214-242.

Boyd IA, Davey MR (1968) Composition of peripheral nerves. Edinburgh: Livingstone.

Diener HC, Dichgans J, Guschlbauer B, Mau H (1984) The significance of proprioception on postural stabilization as assessed by ischemia. Brain Res 296:103-109.

Diener HC, Horak FB, Nashner LM (1988) Influence of stimulus parameters on human postural responses. J Neurophysiol 59:1888-1905.

Do MC, Bussel B, Breniere Y (1990) Influence of plantar cutaneous afferents on early compensatory reactions to forward fall. Exp Brain Res 79:319-324.

Ghez C, Gordon J, Ghilardi MF (1995) Impairments of reaching movements in patients without proprioception. 2. Effects of visual information on accuracy. J Neurophysiol 73:361-372.

Grillner S (1975) Locomotion in vertebrates: central mechanisms and reflex interaction. Physiol Rev 55:247-304.

Hoover DM, Carlton WW (1981) The subacute neurotoxicity of excess pyridoxine $\mathrm{HCl}$ and clioquinol (5-chloro-7-iodo-8-hydroxyquinoline) in beagle dogs. II. Pathology. Vet Pathol 18:757-768.

Horak FB, Macpherson JM (1996) Postural orientation and equilibrium. In: Handbook of physiology, Sec 12, Exercise: regulation and integration of multiple systems (Rowell LB, Shepherd JT, eds), pp 255-292. New York: Oxford UP.

Hulliger M, Bishop GM, Tyson R, Sutherland GR, Banks RW (2000) The effects of deafferentation on gait in the cat: sensorimotor syndrome and recovery. J Physiol (Lond) 525:8S-9S.

Inglis JT, Macpherson JM (1995) Bilateral labyrinthectomy in the cat: effects on the postural response to translation. J Neurophysiol 73:1181-1191.

Inglis JT, Horak FB, Shupert CL, Jones-Rycewicz C (1994) The importance of somatosensory information in triggering and scaling automatic postural responses in humans. Exp Brain Res 101:159-164.

Koshland GF, Smith JL (1989) Mutable and immutable features of pawshake responses after hindlimb deafferentation in the cat. J Neurophysiol 62:162-173.

Krinke G, Heid J, Bittiger H, Hess R (1978) Sensory denervation of the plantar lumbrical muscle spindles in pyridoxine neuropathy. Acta Neuropathol (Berl) 43:213-216.

Larue J, Bard C, Fleury M, Teasdale N, Paillard J, Forget R, Lamarre Y (1995) Is proprioception important for the timing of motor activities? Can J Physiol Pharmacol 73:255-261.

Macpherson JM (1988) Strategies that simplify the control of quadrupedal stance. 2. Electromyographic activity. J Neurophysiol 60:218-231.

Macpherson JM (1994) The force constraint strategy for stance is independent of prior experience. Exp Brain Res 101:397-405.

Macpherson JM, Lywood DW, van Eyken A (1987) A system for the analysis of posture and stance in quadrupeds. J Neurosci Methods 20:73-82.

Nardone A, Tarantola J, Miscio G, Pisano F, Schenone A, Schieppati M (2000) Loss of large-diameter spindle afferent fibers is not detrimental to the control of body sway during upright stance: evidence from neuropathy. Exp Brain Res 135:155-162.

Prochazka A (1996) Proprioceptive feedback and movement regulation. In: Handbook of physiology, Sec 12, Exercise: regulation and integration of multiple systems (Rowell L, Shepherd JT, eds), pp 89-127. New York: Oxford UP.

Sanes JN, Mauritz KH, Dalakas MC, Evarts EV (1985) Motor control in humans with large-fiber sensory neuropathy. Hum Neurobiol 4:101-114.

Schaeppi U, Krinke G (1985) Differential vulnerability of 3 rapidly conducting somatosensory pathways in the dog with vitamin $\mathrm{B}_{6}$ neuropathy. Agents Actions 16:567-579.

Schaumburg H, Kaplan J, Windebank A, Vick N, Rasmus S, Pleasure D, Brown MJ (1983) Sensory neuropathy from pyridoxine abuse. N Engl J Med 309:445-448.

Watt DGD (1976) Responses of cats to sudden falls: an otolithoriginating reflex assisting landing. J Neurophysiol 39:257-265.

Windebank AJ, Low PA, Blexrud MD, Schmelzer JD, Schaumburg HH (1985) Pyridoxine neuropathy in rats: specific degeneration of sensory axons. Neurology 35:1617-1622.

Xu Y, Sladky JT, Brown MJ (1989) Dose-dependent expression of neuronopathy after experimental pyridoxine intoxication. Neurology 39: 1077-1083. 